\title{
FIPSER a novel low cost and high performance readout concept for fast signals
}

\author{
B. Limyansky ${ }^{a}$, B. Reese ${ }^{a}$, J. Cressler ${ }^{b}$, A. N. Otte ${ }^{* a}$, I. Taboada ${ }^{a}$, C. Ulusoy ${ }^{b}$ \\ ${ }^{a}$ School of Physics \& Center for Relativistic Astrophysics, Georgia Institute of Technology, 837 \\ State Street NW, Atlanta, GA 30332-0430, USA \\ ${ }^{b}$ School of Electrical and Computer Engineering, Georgia Institute of Technology, 777 Atlantic \\ Drive, NW, Atlanta, GA 30332-0250, USA \\ E-mail: bttelgatech.edu
}

We propose a novel readout system FIPSER to record signals from detectors with a fixed pulse shape. The primarily targeted applications, but not the only ones, are the digitization of signals from fast photon detectors, i.e. classical photomultipliers and Silicon photomultipliers in astroparticle and high-energy physics experiments. FIPSER is based on the flash analog to digital converter (FADC) concept but has the potential of significantly lower power consumption and costs. These savings are realized by reducing the number of comparators in FIPSER by at least one order of magnitude when compared to an FADC.

In this paper we present first results of a study that investigates the performance of FIPSER in terms of achievable charge and time resolution and compare the results to the Poisson limit, which is often a requirement to be met in experiments.

International Conference on New Photo-detectors, PhotoDet2015

6-9 July 2015

Moscow, Troitsk,Russia

\footnotetext{
* Speaker.
} 


\section{Introduction}

Astroparticle physics has experienced dramatic experimental advancements in the past decade thanks to new generations of instruments in all disciplines. One reason for the recent achievements had been the upscaling of experiments utilizing proven experimental techniques in order to achieve higher sensitivities. In consequence instruments are now much larger and/or have much finer granularity, both of which resulted in a steep increase in the number of signal channels.

Present experiments have thousands of signal channels and the next generation of instruments will have tens if not hundreds of thousands of channels. The massive increase in the number of channels is a challenge when it comes to meeting power and cooling requirements at remote locations not to mention financial constraints. In order to mitigate these boundary conditions several new concepts to digitize signals had been developed that all aim at lowering the per channel costs, and reduce power consumption.

From the point of signal digitization, FADCs are ideal choices but they are costly and consume a non negligible amount of power $(>100 \mathrm{~mW}$ ) even when running at samplings speeds of only a few hundred Megasamples per second and moderate 8-bit resolution. A good alternative that has emerged over the past two decades are switched capacitor arrays (SCAs), e.g. [1]. In an SCA based system, unlike in an FADC system, the analog amplitude is not digitized right away but the analog values are temporarily stored in an array of capacitors and only digitized when needed. The concept of on-demand digitization is a huge power saver but comes with the disadvantage that only short time periods of typically a few microseconds can be digitized.

We propose a different approach called FIPSER (FIxed Pulse Shape Efficient Readout) that retains the FADC concept but allows similar if not larger power and costs savings than the SCA approach. In comparison to SCA systems FIPSER allows the continuous digitization of analog signals without having to stop the sampling. In addition to being truly dead time free, the system opens up new avenues to apply event selection at the detector's trigger level. Another impact of FIPSER on experiments is a significant decrease in data volume.

The proposed FIPSER concept is presented in Section 2. In Section 3, the configuration of FIPSER and the simulations are described that are used to assess the feasibility of the concept. The performance of FIPSER in terms of amplitude and time resolution are discussed in Sections $\emptyset$ and 5. respectively. Conclusions and next steps are discussed in Section 7.

\section{The FIPSER concept}

In an FADC system the input range is discretized into $2^{n}$ levels, where $n$-bit is the resolution of the FADC. A typical realization of an FADC system consists of $2^{n}-1$ comparators with the threshold of each comparator set to one of $2^{n}-1$ non-zero discrete levels. The signal that is to be digitized is fed to the input of all the comparators and the digitized value is then given by the triggered comparator with the highest set threshold level.

The number of comparators is one main factor influencing the power consumption of an FADC system and the produced data volume. Reducing the number of comparators would thus result in significant power savings and data reductions. 


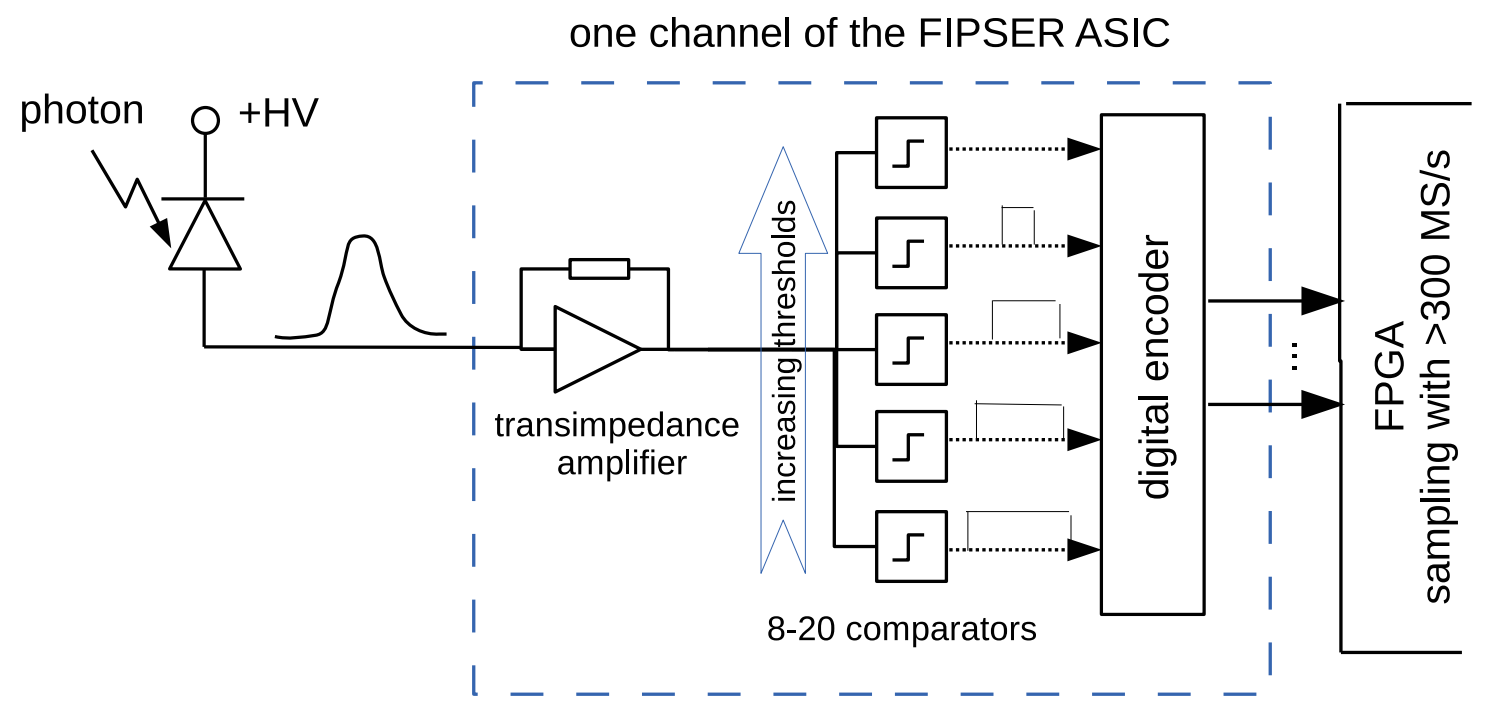

Figure 1: Concept of the FIPSER readout. See text for details.

One possibility to reduce the number of comparators comes from the realization that in many applications the spacing between subsequent discrete levels of the FADC is not required to be the same throughout the dynamic range. While a close spacing is necessary for the digitization of signals with small amplitudes a coarser spacing is sufficient for signals with large amplitudes. The number of comparators and to what levels they are set depends on the expected signal shape and dynamic range. Exploiting this is the idea behind FIPSER.

Figure 1 shows a simplified block diagram of FIPSER. The analog signal is split into ndifferent branches that each lead to the input of a comparator. Each comparator is set to a different threshold value. The output of a comparator is logic high when the input signal is above the comparator's threshold value and logic low otherwise. The comparator signals are fed into an FPGA via an optional digital encoder that reduces the number of logic signal lines if needed. The FPGA records the status of the input signals at a fixed sampling rate and processes the data before they are being recorded on hard disk.

FIPSER can thus be described as an FADC with varying resolution throughout the dynamic range. As we will show in the following a few ten comparators are sufficient to cover a dynamic range of three orders of magnitude.

\section{Configuration of FIPSER}

For this case study we consider a scenario that is a typical one encountered in astroparticle physics, for example, the digitization of a signal produced by Cherenkov-light flashes and recorded with photomultipliers. The statistical nature of the photon generating process sets a natural upper limit on the required resolution of the readout system. When $N$ is the number of detected photoelectrons, the true number of photoelectrons is in the range $N \pm \sqrt{N}$, i.e. the relative uncertainty on the detected signal is $1 / \sqrt{N}$. A requirement on the readout system is thus to allow the recon- 
struction of the amplitude or charge of the recorded signal with an uncertainty that is better then $1 / \sqrt{N}$. We adopt the $1 / \sqrt{N}$ requirement as a benchmark for our study. For the dynamic range we chose 1,000 , where the unit for amplitude is the quantity that is to be reconstructed, for example, the signal equivalent to one photoelectron. A dynamic range of 1,000 is a typical requirement and should allow to easily extrapolate our results to applications with different requirements.

While the requirement on the resolution of the reconstructed number of photoelectrons applies to the majority of experiments in astroparticle physics, an equally general requirement on the reconstruction of time does not exist. We, therefore, refrain from formulating such a requirement. Time resolution and all other time related quantities are reported in units relative to the full width at half maximum (FWHM) of the signal that is to be recorded. In other words, the FWHM of the signal sets the unit of time. The advantage of quoting time in units of the signal width is that it allows for a quick comparison of the simulated FIPSER performance with experimental requirements in various applications without having to redo the simulations. As a consequence of choosing the FWHM as time unit the sampling rate of FIPSER is given in units inverse of the FWHM. For example, a sample rate of two means that FIPSER samples two times per signal FWHM. In these units a sampling rate of one is about the Nyquist limit. In order to properly interpret our results we note that for a detector signal with a FWHM of $10 \mathrm{~ns}$, a time of 0.03 translates to 300 ps and 0.01 to $100 \mathrm{ps}$, respectively. A sampling rate of 3 per FWHM translates to 300 Megasamples per second.

We use a log-normal function as signal shape, which mimics the often encountered case of a fast rising edge and a slower decaying pulse amplitude:

$$
A \cdot \exp \left(-\frac{\ln (t / \tau)^{2}}{2 \sigma^{2}}\right),
$$

with $\tau=7 / 8$, and $\sigma=0.45$. The chosen parameterization yields a FWHM of one. The threshold levels of the $n$ comparators are logarithmically spaced according to

$$
i^{\text {th }} \text { threshold level }=0.25 \cdot 2000^{i /(n-1)},
$$

with $i$ running from 0 to $n-1$. Independent of the number of comparators $n$, the first threshold is always set to 0.25 and the highest threshold is set to 500 . We note that we did not optimize the procedure to set the threshold levels, which probably leaves some room for improvements in the future. A total of four different configuration of FIPSER are tested with $n=8,12,16$, and 20 comparators.

For each FIPSER configuration a total of 8,000 individual signal traces are simulated each with a random signal amplitude $A$ between 1 and 1,000. Cases with and without additional white noise added to the simulated trace are considered and will be discussed in subsequent sections.

The simulated and then digitized signal is reconstructed with two independent methods. The first method is a least square method using the known signal shape as a fit template. The second method is a probabilistic method that utilizes information about the threshold settings of FIPSER and noise characteristics when reconstructing the signal. Both method yield similarly good performance when reconstructing pulse amplitudes and times. A detailed description of both reconstruction methods will be given elsewhere. 

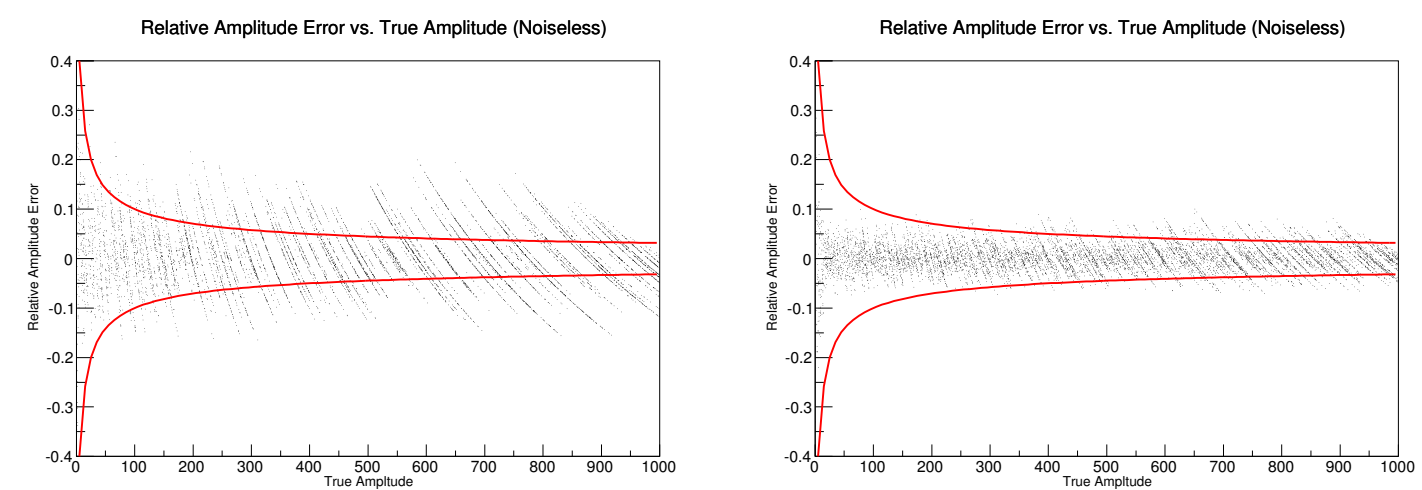

Figure 2: Reconstructed signal amplitudes for two FIPSER configurations with a sampling rate that is three samples per signal FWHM. The left panel shows results for a configuration with 8 comparators and the right panel shows results with 20 comparators. The amplitude reconstruction improves significantly when the number of comparators is increased from eight to twenty. The root mean square values for both configurations are shown in Figure 3.
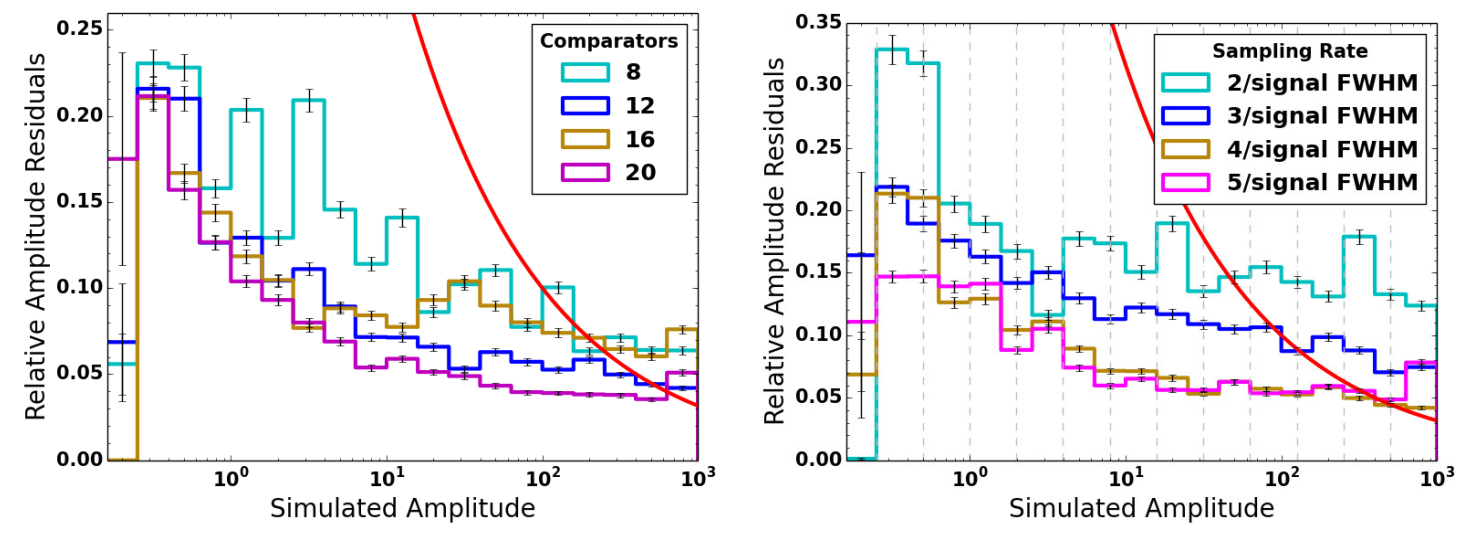

Figure 3: Left panel: Relative amplitude residuals vs. true signal amplitude without noise for four different numbers of comparators at a fixed sampling rate of 4 per FWHM. Right panel: Residuals for four different sampling rates and a fixed number of twelve comparators. The dashed vertical lines show the twelve threshold levels.

\section{Charge resolution}

In order to judge the performance of reconstructing the signal amplitude we calculate the relative amplitude residual $\left(A_{\text {rec }}-A_{\text {true }}\right) / A_{\text {true }}$ between the reconstructed $A_{\text {rec }}$ and simulated amplitude $A_{\text {true }}$ for each simulated and reconstructed signal. Figure 2 shows the scatter plots of the amplitude residuals as a function of $A_{\text {true }}$ for two different numbers of comparators. The red lines in both panels show the Poisson limit, which is met if $68 \%$ of the residuals fall between the lines. While many events are reconstructed outside the limits for a configuration with eight comparators, most of the events are reconstructed within the limits if 20 comparators are used instead of 8 . Both simulations were performed with three samples per FWHM and without electronic or detector noise.

To allow for a direct comparison between different FIPSER configurations and the Poisson 

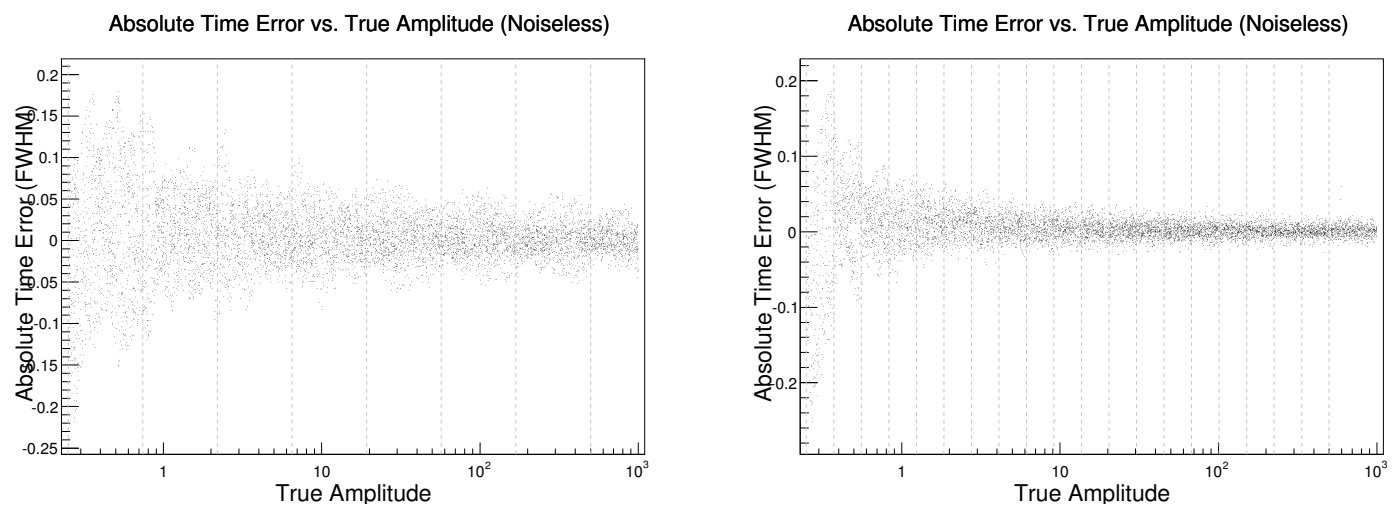

Figure 4: Scatter plots of the differences between the true and reconstructed arrival time for a fixed sampling rate of 3 samples per signal FWHM. Right: 8 comparators, Left: 20 comparators.

limit, the scatter plots are binned in true amplitude and the root mean square (RMS) of the residuals are calculated for each bin. The left panel in Figure 3 shows the calculated RMS values vs. true amplitude for FIPSER configurations with 8, 12, 16, and 20 comparators running at the same sampling rate of 4 per FWHM. As expected the amplitude resolution improves with increasing number of comparators. The Poisson limit requirement is met for configurations with twelve or more comparators everywhere but in the last bin.

The right panel shows the amplitude resolution for a configuration with 12 comparators but different sampling rates. It is interesting to note that the amplitude resolution for a sampling rate of 4 and 5 are comparable.

\section{Time resolution}

The performance to reconstruct time is qualitatively assessed by calculating the difference between the reconstructed and the true time. Figure $\emptyset$ shows scatter plots of the time residuals for a sampling rate of 3 samples per signal FWHM and 8 and 20 comparators, respectively. In both configurations the reconstructed times match the true ones much better than 0.1 FWHM for signal amplitudes larger than 1 . As before we divided the scatter plots in bins of true amplitude and calculate the RMS of the time differences. The results are shown in the left panel of Figure 5 for a sampling rate of 4 per FWHM. The time resolution significantly improves when the number of comparators is increased from 8 to 12 .

The right panel of the same figure shows how for 12 comparators the time resolution improves with increasing sampling rate. While a dramatic improvement is evident when the sampling rate is increased from 2 to 3 , the time resolution does not change much for larger sampling rates.

A FIPSER configuration with 12 comparators and a sampling rate of 3 thus seems to provide a performance that is significantly improved only with many more comparators and much higher sampling rates.

\section{Noise}

How noise affects amplitude and time resolution shows Figure 6 for five noise levels. The 

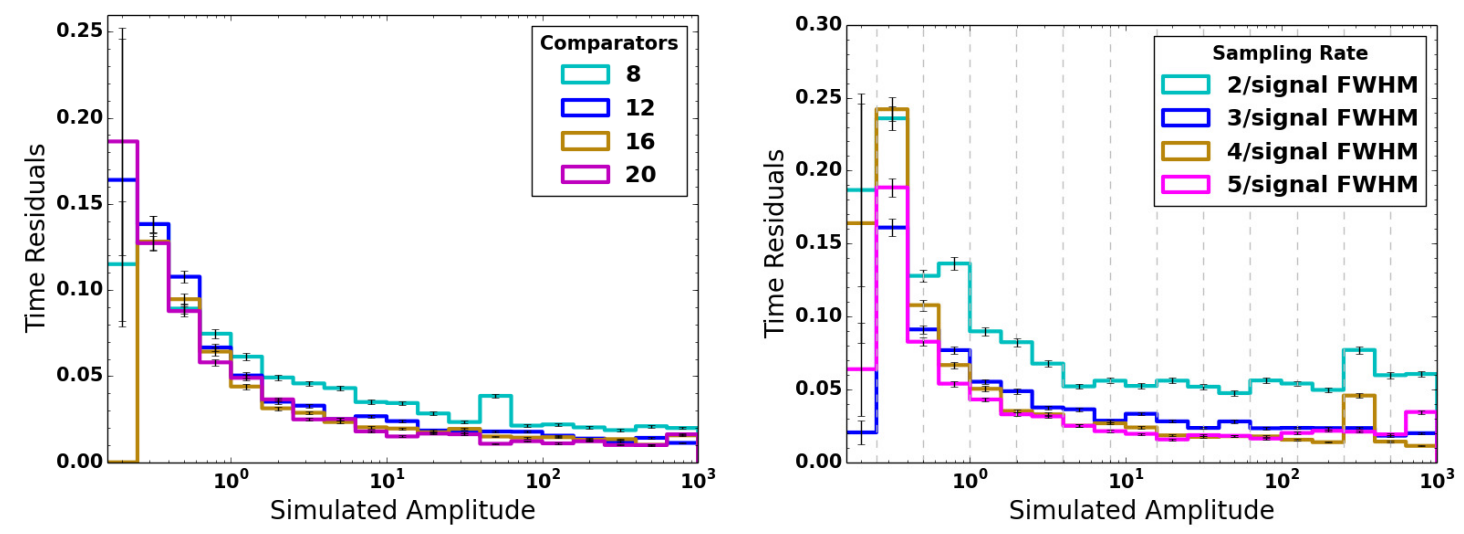

Figure 5: Left panel: Time resolution for a configuration with a fixed sampling rate of 4 and four different number of comparators. Right panel: Time resolution for a configuration with twelve comparators and four different sampling rates. The vertical lines mark the threshold settings.
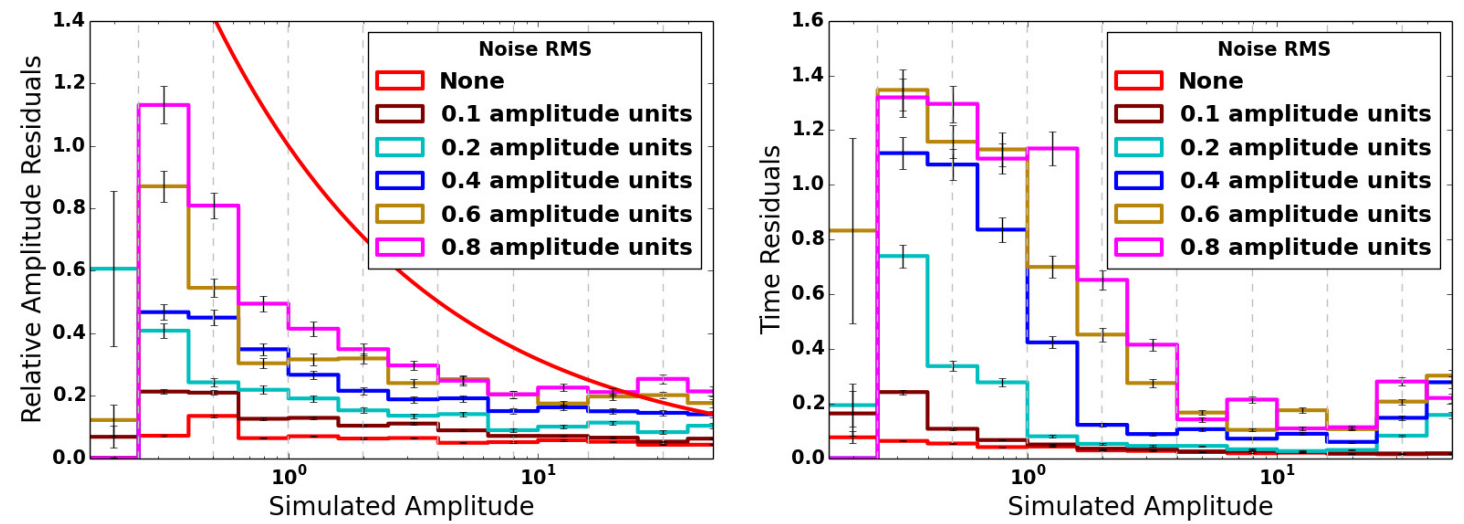

Figure 6: Impact of white noise on the signal reconstruction. Left panel: Root mean square of the relative amplitude residuals for the noiseless case and five different noise levels $0.1,0.2,0.4,0.6$, and 0.8 . The configuration of FIPSER is fixed at a sampling rate of 3 samples per FWHM and 20 comparators. Right panel: Time residuals for the same noise levels and FIPSER configuration.

highest noise level is 0.8 , which can be assumed to be a worst case scenario for most applications. A configuration with 20 comparators is used for the noise study. The dynamic range covered in Figure 6 stops at 50, which is the range where the impact of white noise is most important. From the figure is is evident that the required amplitude resolution is met throughout the dynamic range for all but the two highest noise levels.

\section{Conclusions}

We investigate FIPSER, a novel concept to digitize fast signals with known pulse shapes. The simulation results presented here are very encouraging and show that with 12 comparators and a sampling rates of 3 samples per signal FWHM an amplitude resolution can be achieved that meets the $1 / \sqrt{N}$ requirement over a dynamic range of three orders of magnitude. A time resolution of 
0.05 times the signal FWHM for amplitudes of one seem realistic and improves to about 0.02 for large signal amplitudes.

A limitation of the concept is that the pulse shape needs to be known beforehand. While this should not be a huge problem for most applications, the FIPSER concept needs to be studied in more detail for applications in which pulses of similar amplitudes can overlap frequently. More sophisticated application specific algorithms to reconstruct the signal could mitigate some of the limitations.

Compared to established readout schemes, FIPSER comes with a number of practical advantages:

- Significant power savings due to a large reduction in the number of comparators normally found in digitizers.

- Significant cost savings in the design and fabrication of a readout system.

- Compactness

- Large reduction in data volume

- Dead time free

- Possibility of online event selection and processing

Our next step is the development of an application-specific integrated circuit (ASIC) for FIPSER. The FIPSER ASIC includes an amplifier with low input impedance and shaping for the use with Silicon photomultipliers (SiPMs). The amplifier is especially tailored to handle the large gain and terminal capacitance of SiPMs. First prototypes of the amplifier and comparators have been produced in Silicon-Germanium (SiGe) Heterojunction Bipolar Transistor (HBT) technology and are presently being evaluated.

\section{References}

[1] L. Tibaldo, et al. TARGET: toward a solution for the readout electronics of the Cherenkov Telescope Array, This proceedings. 